\title{
A Meta-Analysis of Eye-Tracking Studies on Text Processing in Children with Reading Disabilities
}

\author{
Jung A Kim, Se Jin Oh, Eunjung Choi, Young Tae Kim, Jee Eun Sung \\ Department of Communication Disorders, Ewha Womans University, Seoul, Korea
}

Correspondence: Young Tae Kim, PhD Department of Communication Disorders, Ewha Womans University, 52 Ewhayeodae-gil, Seodaemun-gu, Seoul 03760, Korea

Tel: $+82-2-3277-2120$

Fax: +82-2-3277-2122

E-mail: youngtae@ewha.ac.kr

Received: July 10, 2018

Revised: August 8, 2018

Accepted: August 8, 2018

This work was supported by the Ministry of Education of the Republic of Korea and the National Research Foundation of Korea (No. NRF2015S1A5A2A03049681)

\begin{abstract}
Objectives: Children with reading disabilities $(\mathrm{RD})$ are at higher risk of becoming scholastic underachievers and having behavioral problems than typical reading children. It is therefore very important to screen for reading disabilities early through overall reading assessments. It is known that eye-tracking tasks are useful for assessing reading ability. This paper reviews studies of eye-tracking and investigates if children with reading problems can be differentiated through eye-tracking tasks. Methods: Twelve studies which met the inclusion criteria were selected from nine electronic databases (Academic Search Complete, PsycINFO, ERIC, PubMed, CINAHL PLUS, ScienceDirect, RISS, DBpia, Kyobo Scholar). A systematic review of literature was done using meta-analysis (Comprehensive Meta-Analysis version 2). Effect sizes were calculated using Hedges' $g$. Results: There was a significant difference in the overall mean effect size between the RD group and the control group. The variables that led to group differences were number of fixations, fixation duration, number of saccades, and number of regressions. However, there was no significant group difference in amplitude of saccades. Conclusion: These results suggest that eye-tracking tasks of text processing are useful tools for discriminating the RD group from the control group and might be used for assessing reading disabilities.
\end{abstract}

Keywords: Eye-tracking, Reading disabilities, Text processing, Meta-analysis
학령기 아동들은 읽기 해독이 가능해지면서 책이나 읽기 자료를 통해 다양한 정보를 얻으며, 이를 바탕으로 학업 성취를 이루거나 사회적 상호작용을 하게 된다. 이처럼 읽기는 일상생활을 영위하는 데 필요한 핵심기술이라고 할 수 있으며(Chung, Kim, \& Yoon, 2017), 높은수준의 인지적 과정으로서 감각 지각, 안구 움직임, 언어 및 의 미적 능력 등을 요구한다. 이러한 능력 중에서 하나라도 결함이 생 기면 읽기장애를 유발할 수 있다(Habib, 2000). 따라서 읽기에 어려 움이 있는 아동들은 일반아동에 비해 학업능력이 떨어지거나 행 동문제를 일으킬 가능성이 더 높다 (Dürrwächter, Sokolov, Reinhard, Klosinski, \& Trauzettel-Klosinski, 2010).

읽기장애(Reading disabilities, RD)는 아동의 연령, 지능, 그리고 교육 정도에 비해 현격하게 낮은 읽기능력을 나타내는 것을 의미한 다. 일반적으로 읽기장애 아동들은 글자를 구별하거나 음소와 글 자를 연결하는 데 어려움을 나타낸다. 아동의 읽기장애 여부는 읽 기교육이 본격적으로 이뤼지는 유치원 이후나 학령기 초반이 되어
서야 평가되는 경향이 있다(Rommelse, Van der Stigchel, \& Sergeant, 2008).

읽기능력을 평가하는 방법은 다양하지만 그 중에서도 읽기와 관 련된 인지적 과정을 분석하기 위해 시선추적장치(eye-tracker)를 사용하여 눈의 움직임을 측정하는 많은 연구들이 수행되어 왔다. 시선추적장치는 피실험자가 글을 읽을 때 사람이 지각하지 못하는 적외선을 눈에 투사하여 수정체에서 반사되는 신호를 통해 시선의 움직임을 읽어내는 전문적인 실험기구이다(Suh, Kim, Pyeon, \& Shin, 2016). De Luca, Borrelli, Judica, Spinelli와 Zoccolotti (2002) 는 시선추적장치를 통해 눈의 움직임을 측정하는 것이 발달적 읽 기 결함을 연구하기 위한 효과적인 방법이 될 수 있다고 했으며, Pavlidis (1981)도 시선추적 과제가 읽기장애 아동 집단과 일반아 동 집단을 구별하는 데 유용하다고 했다. 시선추적 과제를 통해 측 정할 수 있는 변수는 고정(fixation), 도약(saccade), 회귀(regression) 등이다. 고정이란 0.2 초 이상 응시를 할 경우에 일어나며, 도약은 고 
정과 고정 간에 발생하는 시선의 움직임을 의미한다. 회귀는 도약이 오른쪽에서 왼쪽으로 일어나는 경우를 말한다(Lee \& Suh, 2013; Rayner, 1978). 일반적으로 읽기장애 아동은 일반아동에 비해 고정 시간이 더 길고, 도약의 수가 더 많으며, 도약 진폭이 더 짧고, 회귀 도 더 자주 일어나는 특성을 나타낸다(Dürrwächter et al., 2010). Pavlidis (1981)에 의하면 읽기장애 아동들에게서 회귀와 안정적이 지 못한 고정이 더 많이 나타난다고 했다. Rayner (1985)도 읽기장 애 아동들이 일반아동들에 비해 고정 지속시간이 더 길고, 진폭이 작은 도약이 빈번하게 일어난다고 했다. 또한 국가별로 읽기장애 아 동들에 대한 다양한 연구결과를 살펴보면 다음과 같다. 이탈리아 의 읽기장애 아동들은 지속시간이 긴 고정을 빈번하게 나타냈으며 (De Luca, Di Pace, Judica, Spinelli, \& Zoccolotti, 1999). 독일의 경 우에는 더 많은 고정과 짧은 지속시간을 나타낸다고 했다(Hutzler \& Wimmer, 2004). 그리스의 읽기장애 아동들은 좀 더 많은 고정 과 긴 지속시간을 나타냈다(Hatzidaki, Gianneli, Petrakis, Makaronas, \& Aslanides, 2011).

시선추적 연구에서 읽기장애 집단이 나타내는 비전형적인 눈의 움직임을 두고 읽기문제의 원인을 크게 두 가지로 분석하는 경향 이 있다(De Luca et al., 1999; Hatzidaki et al., 2011; Martos \& Vila, 1990; Prado, Dubois, \& Valdois, 2007). 첫째, 언어적 측면에서 음운 이나 어휘의 처리, 읽기 자체의 결함으로 읽기장애가 유발된다고 본다. 둘째, 시각적 정보처리의 측면에서 안구운동의 결함이나 주 의집중력의 부족으로 읽기문제가 야기된다고 본다. 이에 따라 시선 추적 과제의 유형도 언어적 과제와 시각적(비언어적) 과제로 대별 된다. 언어적 과제에서는 주로 단어나 비단어, 문장이나 텍스트 수 준의 자극을 제시하고 묵음이나 음독에 의해 읽게 하며, 시각적 과 제에서는 특정 모양이나 알파벳, 점 등을 제시하여 이들을 찾거나 추적하도록 한다. 대체로 언어적 과제의 경우 읽기장애 아동 집단 과 일반아동 집단이 시선추적 변수(고정, 도약, 회귀 등)에 따라 유 의한 차이를 나타냈다는 연구들이 많은 편이지만 시각적 과제에 대해서는 읽기 측정상의 타당성 문제와 집단 간의 차이가 유의하 지 않다는 연구결과들로 인해 논란의 여지가 있다고 한다(De Luca et al., 1999; Prado et al., 2007).

시선추적 연구를 언어적 과제로 하는 경우, 낱말보다는 문장이 나 텍스트 수준에서 측정하면 여러 가지 장점이 있다(Kaakinen \& Hyönä, 2007; Raney, Campbell, \& Bovee, 2014; Rayner, 1978). 첫 째, 눈의 움직임을 계속적으로 추적함으로써 읽기 수행능력을 실 시간으로 기록할 수 있도록 해 주며, 낱말이나 구, 문장, 텍스트 수 준에서 상호 간의 관련성을 볼 수 있어 전반적인 텍스트 처리능력 을 평가할 수 있도록 해준다. 둘째, 낱말 수준의 과제에 비해 눈의
움직임의 다양한 측면을 분석할 수 있다. 즉, 고정 지속시간, 도약 길 이, 회기 빈도 등과 같이 시선추적 과제를 통해 측정할 수 있는 여러 가지 변수들을 광범위하게 살펴볼 수 있게 해 준다. 셋째, 읽기과제 를 수행하는 동안 눈의 움직임은 자연스러운 것으로서 추가적인 실험통제나 별도의 과제가 요구되지 않는다. 넷째, 눈의 움직임은 피험자의 개별 특성을 반영해주기 때문에 연령, 읽기능력, 주제에 관한 배경지식 등에 따라 다양한 수행능력을 측정할 수 있다.

이와 같이 시선추적 연구가 읽기 과정에서의 반응을 평가하는 데 유용한 방법으로 알려짐에 따라 국내에서도 교육학, 심리학 등 의 분야를 중심으로 상당수의 연구들이 진행되어 왔다. 그러나 이 러한 연구들은 대부분 읽기에 어려움이 없는 일반아동이나 청소 년, 성인들을 대상으로 읽기 특성을 분석하였고(Choi, 2013; Choi, Lowder, Ferreira, Swaab, \& Henderson, 2017; Kwon \& Sturt, 2014; Lee \& Suh, 2013; Suh et al., 2016), 읽기문제를 가진 장애군을 대상 으로 한 연구는 거의 없다. 또한 시선추적 연구들에 대한 메타분석 연구도 거의 없는 실정이다. 따라서 본 연구에서는 시선추적 과제 가 읽기장애 아동 집단과 일반아동 집단의 텍스트 수준에서의 읽 기 특성을 변별하여 나타낼 수 있는지를 살펴보고, 시선추적 과제 를 통해 측정할 수 있는 변수들 중에서 특히 어떤 변수가 집단 간에 유의한 차이를 보이는지를 분석하고자 하였다. 이에 따른 연구문 제는 다음과같다.

1. 읽기장애 아동과 일반아동이 텍스트 처리 특성에서 유의한 차이를 나타내는가?

2. 읽기장애 아동과 일반아동이 시선추적 연구의 주요 측정치에 서 유의한 차이를 나타내는가?

1) 고정(fixation)에서 유의한 차이를 나타내는가?

2) 도약(saccade)에서 유의한 차이를 나타내는가?

3) 회귀(regression)에서 유의한 차이를 나타내는가?

\section{연구방법}

\section{문헌검색}

시선추적장치를 활용한 읽기장애 아동들의 텍스트 이해의 특징 을 알아보기 위해 2018년 6월 18일에 국내외 데이터베이스와 관련 논문의 참고문헌을 통해 관련 논문들을 수집하였다. 국내(DBpia, RISS, Kyobo Scholar) 및 국외(Academic Search Complete, PsycINFO, ERIC, PubMed, CINAHL Plus, ScienceDirect) 9 개의 데이 터베이스에서 검색어("eye track*" OR eye-track* OR "eye movement") AND reading AND (dyslexia OR dyslexic OR "reading disability" OR "reading disorder" OR "reading problem" OR "poor 
reader”)를 활용하여 검색을 실시하였다.

\section{논문의 선정 기준}

문헌 검색을 통해 Academic Search Complete에서 102편, PsycINFO에서 157편, ERIC에서 36편, PubMed에서 137편, CINAHL Plus에서 32편, ScienceDirect에서 33편, RISS, DBpia, Kyobo Schol$\operatorname{ar}$ 에서 0 편으로 총 497 개의 논문이 검색되었다. 이 중 full-text 이 용 가능 여부, peer reviewed journal, 영어로 작성된 논문을 기준으 로 284편을 선정하였으며, 중복된 논문 123 편을 제외한 161 편의 논 문과 수작업 검색 논문을 대상으로 포함 및 제외기준을 적용하여 총 12 편의 논문을 분석 대상 논문으로 선정하였다. 분석 대상 논문 의 포함 기준으로는 (1) 학령기 읽기장애 아동을 대상으로 한 연구,
(2) 읽기장애 아동과 연령이 통제된 일반아동을 비교한 집단 비교 연구, (3) 시선추적장치를 사용하여 종속변수의 값을 제시한 연구, (4) 텍스트 수준의 읽기과제를 수행한 연구를 분석대상 논문으로 포함하였으며, 이 모든 기준에 부합되지 못한 연구들은 분석대상 에서 제외하였다. 논문 선정 과정에 대한 순서도와 선정 및 제외 기 준은 각각 Figure 1과 Table 1에 제시하였다. 또한 선정된 개별 논문 의 특성은 Appendix 1에 제시하였다.

\section{자료의 분석}

\section{자료의 코딩 및 분석}

연구자, 게재연도, 실험집단 정보 및 표본 크기, 과제 종류, 종속 변수 종류 등을 데이터로 입력하였다. 독립변인은 집단이며, 종속
Inclusion criteria

1. Participants: school-aged children with dyslexia and their age-matched controls 2. Measurement eye-tracking technique 3. Design: group comparison study

4. Task: text reading

5. Outcome measures: number of fixations, fixation duration, number of saccades, amplitude of saccades, number of regressions

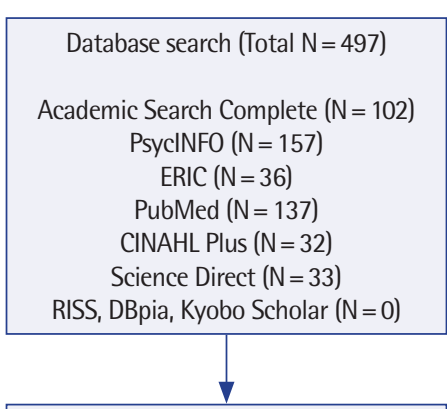

Records filtered based on full text, peer reviewed journal, Engligh

$$
(\mathrm{N}=284)
$$

After duplication removed $(\mathrm{N}=161)$

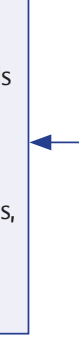

Exclusion criteria

1. Participants: infants, adults, children with other disorders

2. Measurement: other techniques

3. Design: not group comparison, case studies, review articles

4. Task: word reading, non-linguistic tasks

5. Outcome measures: non-relevant measures

Studies included from hand search $(\mathrm{N}=2)$

Figure 1. Flowchart of studies included from search.

Table 1. Criteria for inclusion and exclusion

\begin{tabular}{lll}
\hline & \multicolumn{1}{c}{ Inclusion } & Exclusion \\
\hline Participants & School-aged children with dyslexia and their age-matched controls & Infants, adults, children with other disorders \\
Measurement & Eye-tracking technique & Other techniques \\
Design & Group comparison studies & Case studies, review articles \\
Task & Text reading & Word reading, non-linguistic tasks \\
Outcome measures & Number of fixations, fixation duration, number of saccades, & Non-relevant measures \\
& amplitude of saccades, number of regressions & \\
\hline
\end{tabular}


Jung A Kim, et al. • A Meta-Analysis of Text Processing in Children with Reading Disabilities

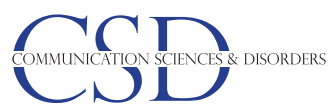

변인은 시선추적 관련 측정치였다. 각 종속변수에 대한 효과 크기 는 선정된 연구에서 평균과 표준편차가 제시된 경우 그 값을 사용 하였고, 평균과 표준편차가 제시되지 않은 경우, $p$ 값을 사용하여 효과크기를 산출하였다. 읽기장애 아동과 일반아동의 읽기 시 시 선추적의 특징을 분석하기 위해 시선추적 연구의 주요 측정치인 고 정 수(number of fixations), 고정 지속시간(fixation duration), 도약 수(number of saccades), 도약 진폭(amplitude of saccades), 회귀 수 (number of regressions)에 대하여 효과크기를 산출하여 시선추적 측정치의 유형별 효과크기를 알아보고자 하였다.

메타분석은 Comprehensive Meta-Analysis (CMA 2.0)를 사용 하여 분석하였다. 각 과제에 따른 연구별 효과크기는 교정한 평균

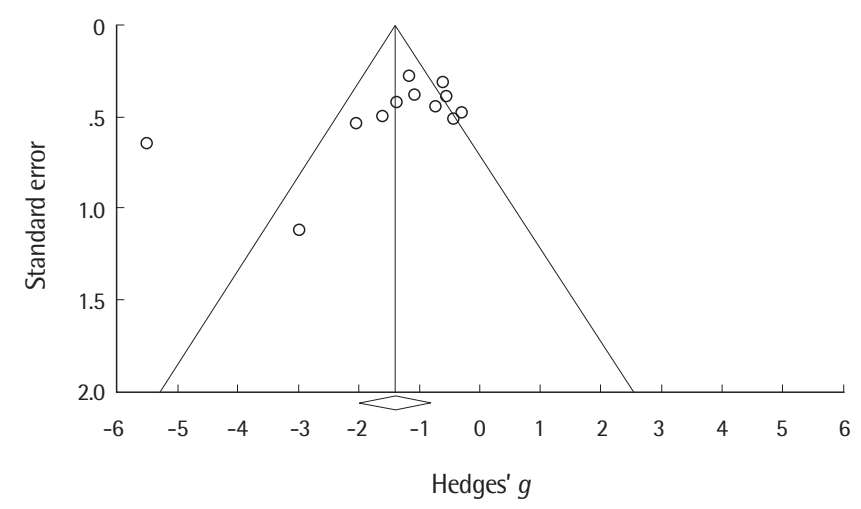

Figure 2. Funnel plot of standard error by Hedges' $g$.
차이인 Hedges' $g$ 를 산출하였으며, $95 \%$ 신뢰구간 및 유의수준 .05 를 기준으로 효과크기의 유의성을 검증하였다. 또한 본 연구에 포 함된 연구들의 집단 및 방법 등의 이질성을 고려하여 무선효과모형 으로 결과값을 제시하였다.

대상 연구들의 질 평가

본 연구의 분석대상으로 선정된 논문들의 질적 평가를 위하여 Gersten 등(2005)의 필수 질적 지표(Essential Quality Indicators) 를 사용하였다. 이 척도에서는 연구대상자에 대한 정보, 연구방법 과 통제 집단에 대한 설명, 연구에서의 측정 변인, 그리고 데이터 분 석과 관련한 항목들에 대해 3점 척도로 평가한다. 본 연구에 포함 된 총 12 개 연구 모두의 질적 수준을 평가한 결과, 연구방법 관련 지표를 제외한 나머지 지표들은 모두 평균 3점으로 나타났다. Kirkby, Blythe, Drieghe와 Liversedge (2011)과 Martos와 Vila (1990)의 연구에서는 연구방법에서 읽기과제의 수준을 구체적으로 명시하 지 않아각각 평균 2.5 점으로 평가되었다.

Table 2. Effect size of overall outcome measures between RD and control groups

\begin{tabular}{lccc}
\hline & Hedges' $g$ & $95 \% \mathrm{Cl}$ & $p$-value \\
\hline Overall & -1.391 & -1.993 to -.789 & .000 \\
\hline
\end{tabular}

$\mathrm{RD}=$ reading disability.
Study name

\begin{tabular}{|c|c|c|c|c|c|c|c|c|}
\hline & & $\begin{array}{c}\text { Hedges's } \\
g\end{array}$ & $\begin{array}{l}\text { Standard } \\
\text { error }\end{array}$ & Variance & $\begin{array}{l}\text { Lower } \\
\text { limit }\end{array}$ & $\begin{array}{l}\text { Upper } \\
\text { li mit }\end{array}$ & 2-Value & p.Value \\
\hline Bucci et al. (2012) & Combined & -1.599 & 0.496 & 0.246 & -2.571 & -0.628 & -3.227 & 0.001 \\
\hline De Luca et al. (1999) & Combined & -0.422 & 0.509 & 0.259 & -1.419 & 0.575 & -0.829 & 0.407 \\
\hline Hatzidaki et al. (2011) & Combined & -0.538 & 0.389 & 0.151 & -1.300 & 0.224 & -1.384 & 0.166 \\
\hline \multicolumn{2}{|c|}{ Hutzler \& Wimmer (2004) Combined } & -2.049 & 0.534 & 0.285 & -3.096 & -1.002 & -3.836 & 0.000 \\
\hline Hyona \& Olson (1995) & Combined & $\cdot 0.609$ & 0.310 & 0.096 & -1.217 & -0.002 & -1.965 & 0.049 \\
\hline Kirkby et al. (2011) & Combined & -0.288 & 0.478 & 0.229 & -1.225 & 0.650 & -0.601 & 0.548 \\
\hline Martos \& Vla (1990) & Combined & -1.171 & 0.278 & 0.077 & -1.715 & -0.626 & .4 .214 & 0.000 \\
\hline Prado et al. (2007) & Combined & -1.361 & 0.422 & 0.178 & -2.187 & -0.534 & -3.227 & 0.001 \\
\hline Razuk et al. (2018) & Combined & -1.077 & 0.382 & 0.146 & -1.827 & -0.328 & -2.817 & 0.005 \\
\hline Seassau et al. (2014) & Combined & -5.496 & 0.648 & 0.420 & .6 .766 & .4 .227 & -8.484 & 0.000 \\
\hline Trauzettel et al. (2010) & Combined & -2.978 & 1.122 & 1.259 & -5.178 & -0.779 & -2.654 & 0.008 \\
\hline \multirow[t]{2}{*}{ Vagge et al. (2015) } & Combined & -0.727 & 0.444 & 0.197 & -1.597 & 0.144 & -1.636 & 0.102 \\
\hline & & -1.391 & 0.307 & 0.094 & -1.993 & -0.789 & -4.528 & 0.000 \\
\hline
\end{tabular}

Hedges's $g$ and $95 \% \mathrm{Cl}$

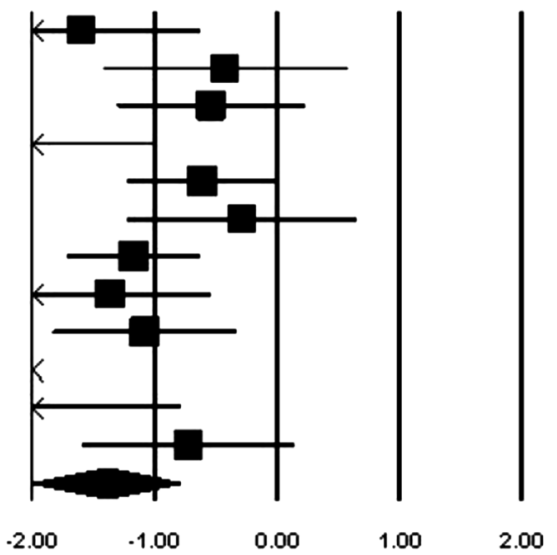

Favours A

Figure 3. Forest plot of overall outcome measures between reading disability (RD) and control groups. 


\section{출판편향 검증}

포함된 연구들의 출판편향 오류를 검증하기 위해 funnel plot를 통해 좌우대칭성을 시각적으로 확인한 결과(Figure 2), 소수의 연구 들이 비대칭성을 유발하는 것으로 관찰되어 비대칭성에 대한 통계 적 분석을 위해 Egger의 회귀분석을 실시하였다. 분석 결과, bias= $-3.98(t=1.86, d f=10, p>.05)$ 로 나타나, 본 연구에 포함된 연구들에 대한 출판편향은 통계적으로 유의하지 않음을 확인하였다.

\section{신뢰도 평가}

언어병리학 박사과정 연구자 3 인이 분석에 포함된 12 편의 논문 에 대하여 각 논문의 정보 및 통계값을 코딩하여 효과크기를 산출 한 후 항목별 결과를 비교한 결과, 평가자 간 신뢰도는 $100 \%$ 였다.

\section{연구결과}

\section{연구의 질적 평가}

연구대상

본 연구의 분석대상으로 채택된 12 개의 논문은 모두 학령기 아 동을 대상으로 하였다. 총 408명의 대상자 중 읽기장애 아동은 207 명으로 평균연령은 11.35 세, 통제 집단인 일반아동은 201명으로 평균연령은 10.75 세였다. 각 연구별로 대상자들의 모국어가 다양 했는데, 대상자의 모국어는 불어(4편), 이탈리아어(2편), 그리스어 (1편), 독일어(2편), 영어(2편), 그리고 스페인어(1편)로 총 6가지의 언어였다.

\section{연구과제}

연구과제는 모두 텍스트 읽기인데, 텍스트의 길이는 최소 30 단어 에서 최대 212단어였다. 모든 연구에서 대상 아동들은 텍스트를 음 독하거나 묵독하여 과제를 수행했고, 읽기 과정 동안 시선추적장 치를 통한 측정이 이루어졌다.

\section{메타분석 결과}

\section{텍스트 처리 특성}

읽기장애 아동과 일반아동의 텍스트 처리 특성을 분석한 결과, $g=-1.391, p<.001,95 \%$ 신뢰구간(-1.993, -.789)으로 두 집단이 텍 스트 처리 특성에서 유의미한 차이를 보이는 것으로 나타났다. 메 타분석 결과는 Table 2와 Figure 3에 제시하였다.

\section{주요 측정치별 특성}

읽기장애 아동과 일반아동의 텍스트 읽기 특성을 살펴보기 위 해 시선추적 연구의 세 가지 주요 측정치를 분석하였다. 각 주요 측

Table 3. Effect size of sub-measures between $\mathrm{RD}$ and control groups

\begin{tabular}{lccc}
\hline & Hedges' $g$ & $95 \% \mathrm{Cl}$ & $p$-value \\
\hline Number of fixations & -2.733 & -4.180 to -1.286 & .000 \\
Fixation duration & -3.393 & -5.151 to -1.634 & .000 \\
Number of saccades & -2.948 & -4.618 to -1.278 & .001 \\
Amplitude of saccades & 1.997 & -.349 to 4.344 & .096 \\
Number of regressions & -2.028 & -3.361 to -.694 & .003
\end{tabular}

$\mathrm{RD}=$ reading disability.

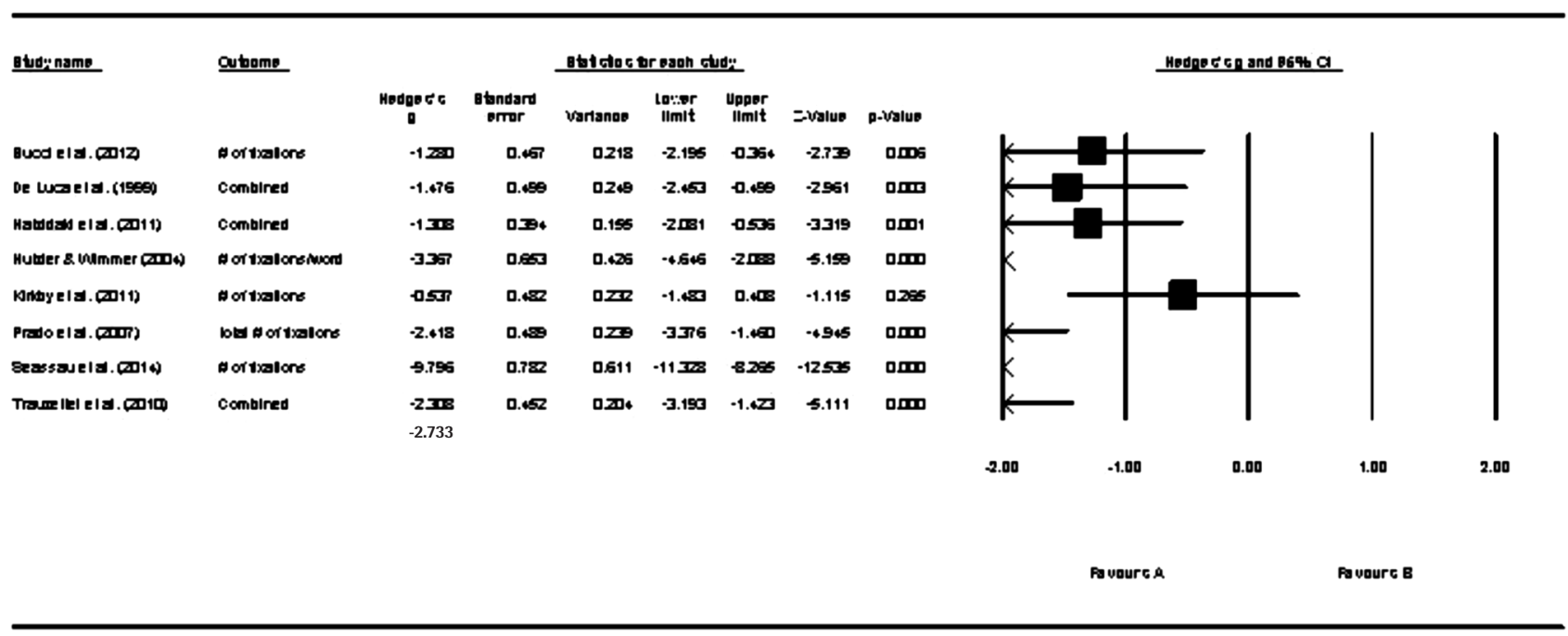

Figure 4. Forest plot of the number of fixations between reading disability (RD) and control groups. 
정치에 대한 효과크기의 분석결과는 Table 3에 제시하였다.

두 집단 간의 고정을 고정 수와 지속시간에 의해 비교한 결과, 고 정 수는 $g=-2.733, p<.001,95 \%$ 신뢰구간(-4.180, -1.286)으로 유의 한 차이를 보였다(Figure 4). 또한 고정 지속시간에서도 $g=-3.393$, $p<.001,95 \%$ 신뢰구간(-5.151, -1.634)으로 두 집단 간에 유의한 차 이가 있었다(Figure 5). 즉, 읽기장애 아동 집단이 일반아동 집단에 비해 유의하게 더 많은 고정 수와 더 긴 고정 지속시간을 보인 것으 로 나타났다.
두 집단 간의 도약을 도약 수와 진폭에 의해 비교한 결과, 도약수 는 $g=-2.948, p<.01,95 \%$ 신뢰구간(-4.618, -1.278)으로 유의한 차 이를 보였다(Figure 6). 즉, 읽기장애 아동 집단이 일반아동 집단에 비해 유의하게 더 많은 도약 수를 보인 것으로 나타났다. 도약 진폭 은 도약의 정확성을 판단하는 측정치로 안구 도약이 일어난 거리에 서 나타난 주사선의 각도나 시간으로 측정된다. 본 연구에서 도약 진폭은 $g=1.997, p>.05,95 \%$ 신뢰구간(-.349, 4.344)으로 두 집단 간에 유의한 차이가 나타나지 않았다(Figure 7).

\begin{tabular}{|c|c|c|c|c|c|c|c|c|c|c|c|c|c|}
\hline \multirow[t]{2}{*}{ Study name } & \multirow[t]{2}{*}{ Outcome } & \multicolumn{7}{|c|}{ Statistics for each study } & \multicolumn{5}{|c|}{ Hedges's $\mathrm{g}$ and $95 \% \mathrm{Cl}$} \\
\hline & & Hedges's & $\begin{array}{l}\text { Standard } \\
\text { error }\end{array}$ & Variance & $\begin{array}{l}\text { Lower } \\
\text { limit }\end{array}$ & $\begin{array}{l}\text { Upper } \\
\text { limit }\end{array}$ & 2-Value & -Value & & & & & \\
\hline B 1001 etal. $(712)$ & toatoi duration & -164 & 0.196 & 024 & -2.611 & $-0.0 \pi$ & -3.301 & ami & & & & & \\
\hline Hatertakiletal. (ه1) & matton durator & $-1, \mathbf{3 6}$ & 0,396 & 0.185 & -2531 & $-0,506$ & -3319 & omi & & & & & \\
\hline Hutter \& Wummer (ang) & 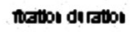 & -1.747 & 0.487 & 0238 & -2.700 & -0.791 & -3.500 & m & & & & & \\
\hline Mmopetal. (D1D) & 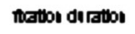 & -0.105 & 0.473 & 0224 & -1505 & 0819 & -0228 & 083 & & & & & \\
\hline Praso etal. (דل) & 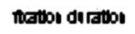 & -1.104 & 0,395 & 0.156 & -1879 & $-0,329$ & -2.791 & oms & & & & & \\
\hline Rarkk etal. (D19) & 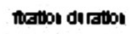 & -0812 & 0.30 & $0.13 \pi$ & -1.58 & -0586 & -2.193 & anss & & & & & \\
\hline Seaszan etal. (D19) & 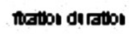 & -9.124 & 0.732 & 0.556 & -10.559 & $-7 \infty 6$ & -12.464 & om & k & & & & \\
\hline \multirow[t]{4}{*}{ Trauzeteletal. (2010) } & combined & $-21,58$ & 2.72 & 8.410 & -25913 & -16242 & $\begin{array}{l}-392 \pi \\
\end{array}$ & om & $k$ & & & & \\
\hline & & $-3,330$ & 0898 & 0.055 & -5.151 & -16006 & -3.781 & om & $k$ & & & & \\
\hline & & & & & & & & & -2.00 & -1.00 & 0.00 & 1.00 & 2.00 \\
\hline & & & & & & & & & & Favours \& & & Favours $B$ & \\
\hline
\end{tabular}

Figure 5. Forest plot of fixation duration between reading disability $(\mathrm{RD})$ and control groups.

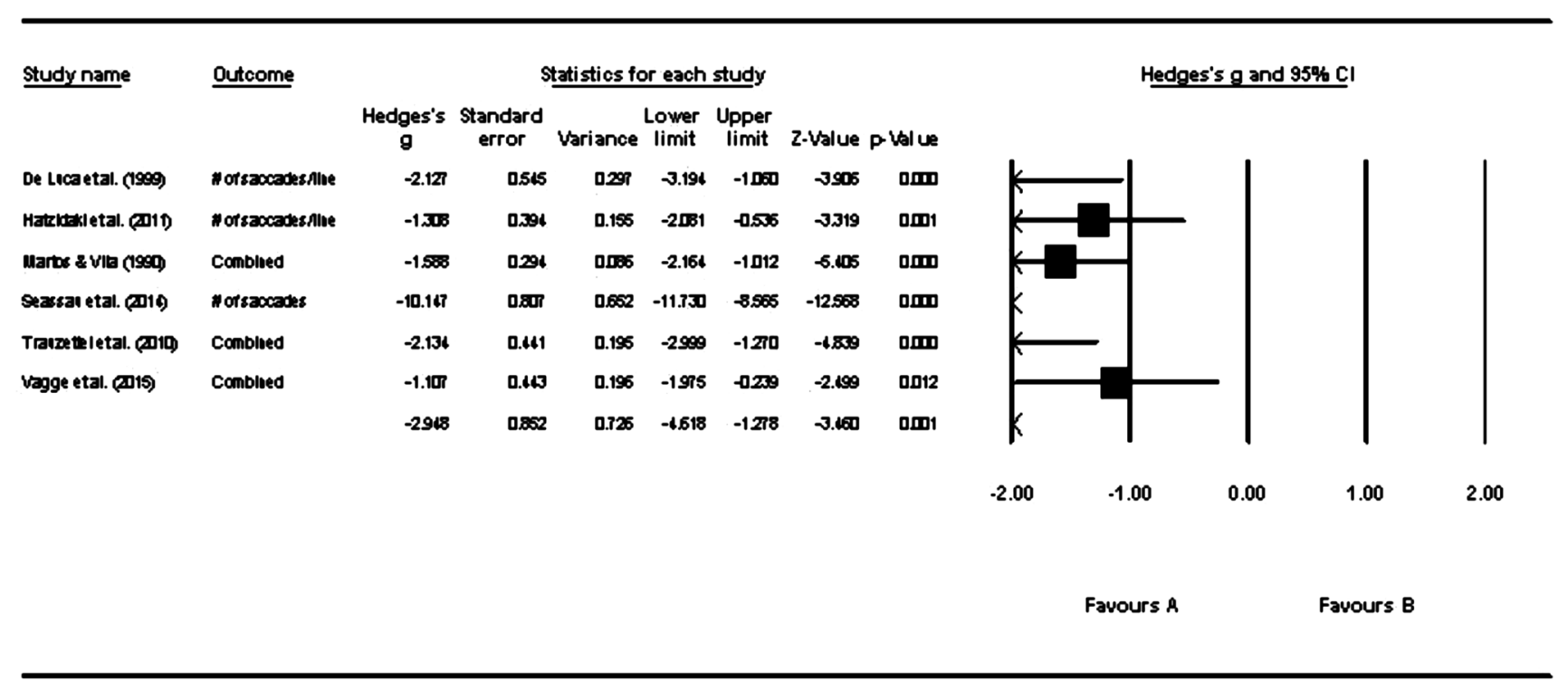

Figure 6. Forest plot of the number of saccades between reading disability (RD) and control groups. 


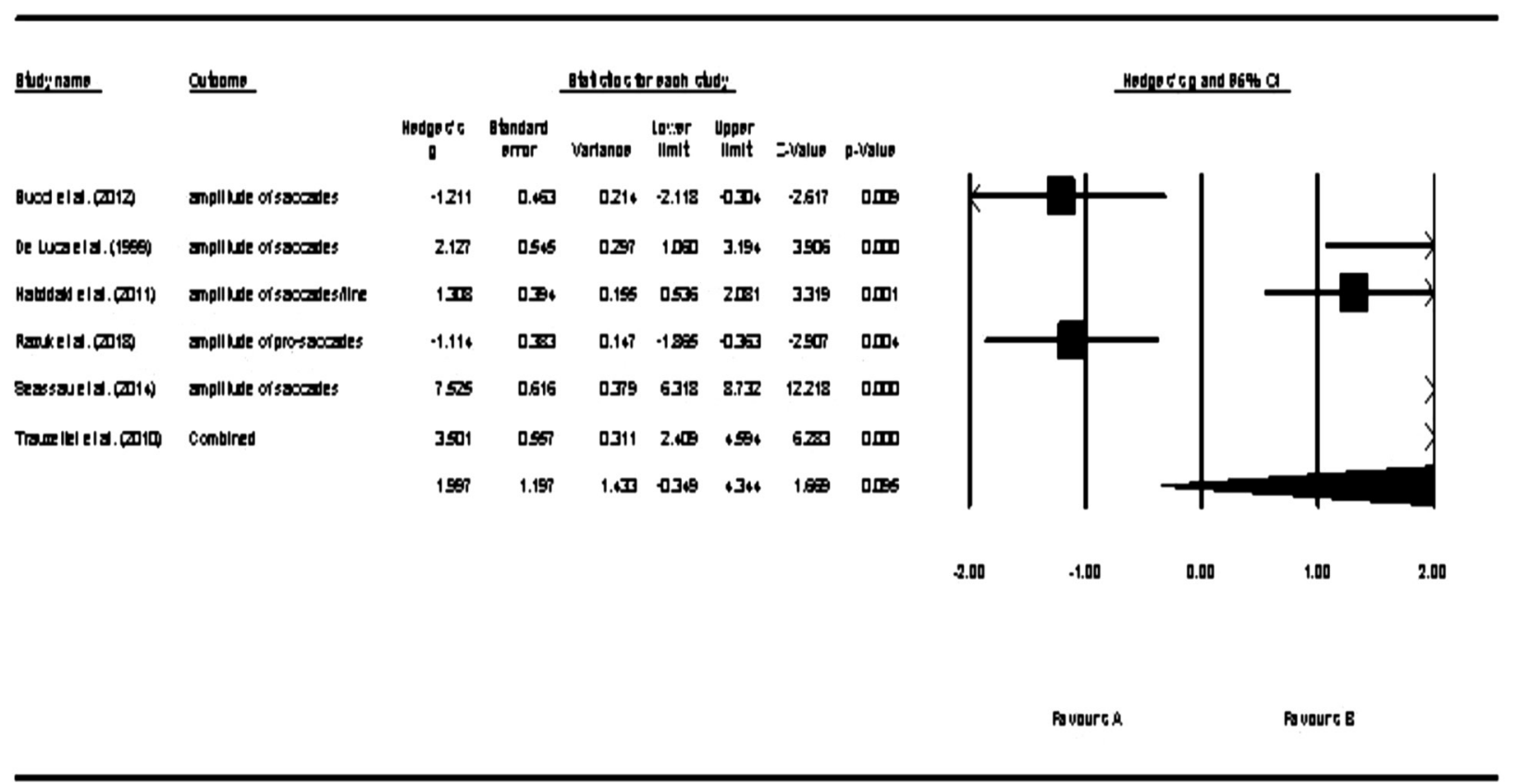

Figure 7. Forest plot of the amplitude of saccades between reading disability (RD) and control groups.

\begin{tabular}{|c|c|c|c|c|c|c|c|c|c|c|c|c|c|}
\hline \multirow[t]{2}{*}{ Study name } & \multirow[t]{2}{*}{ Outcome } & \multicolumn{7}{|c|}{ Statistics for each study } & \multicolumn{5}{|c|}{ Hedges's $\mathrm{g}$ and $9 \mathrm{F \%} \mathrm{Cl}$} \\
\hline & & $\begin{array}{c}\text { Hedges's } \\
g\end{array}$ & $\begin{array}{c}\text { Standard } \\
\text { error }\end{array}$ & Variance & $\begin{array}{l}\text { Lower } \\
\text { limit }\end{array}$ & $\begin{array}{l}\text { Upper } \\
\text { limit }\end{array}$ & 2. value & p.value & & & & & \\
\hline De LIçetal. (1999) & "Oor regress bossallee & -1230 & 0.671 & 0221 & -2155 & -0.311 & -2.600 & $\mathrm{amg}$ & & & & 1 & \\
\hline Hatritaletal. (Di1) & "Nof regress bisnlibe & -1.15 & 0.306 & 0.10 & -1.914 & -0.00 & -2997 & Sח & & & & & \\
\hline Marts \& Vil (1990) & Combleed & $-0,8$ & 0.288 & 0072 & -1.112 & $-0,303$ & 3317 & omi & & & & & \\
\hline Seassal etal. (D19) & \# of regress bis & -6.162 & 0.519 & 0270 & -7.190 & 5.14 & -11288 & om & $k$ & & & & \\
\hline Traizeteletal. (010) & Comblied & $-1 / 81$ & 0.005 & 0.165 & -2 เा & -0.876 & -6.119 & om & & & & & \\
\hline \multirow[t]{4}{*}{ vagge etal. (D19) } & \#of regress bis & -1224 & 0.600 & 0202 & -2105 & $-0,313$ & -2.72 & $0 \mathrm{~m} 5$ & & & & & \\
\hline & & -20208 & 0.000 & Dגब & -7351 & $-0,096$ & -2900 & (IIIJ & $k$ & & & & \\
\hline & & & & & & & & & .2 .00 & .1 .00 & 0.00 & 1.00 & 2.00 \\
\hline & & & & & & & & & & Favours $\mathrm{A}$ & & Favours B & \\
\hline
\end{tabular}

Figure 8. Forest plot of the number of regressions between reading disability (RD) and control groups.

\section{두 집단 간의 회귀를 비교한 결과, 회귀 수는 $g=-2.028, p<.01, \quad$ 논의 및 결론} 95\% 신뢰구간(-3.361, -.694)으로 유의한 차이를 보였다(Figure 8). 즉, 읽기장애 아동 집단이 일반아동 집단에 비해 유의하게 더 많은 회귀 수를 보인 것으로 나타났다.

본 연구는 시선추적장치를 활용한 텍스트 수준에서의 읽기 평가 가 읽기장애 아동과 일반아동을 유의하게 변별하는지를 살펴보 
고, 시선추적 과제의 측정치 중에서 집단 간에 유의한 차이를 나타 내는 측정치를 확인하기 위하여 메타분석을 실시하였다. 문헌 연구 의 포함 및 제외 기준에 따라 12 개의 연구를 분석한 결과, 읽기장애 아동과 일반아동이 텍스트 처리 특성에서 유의미한 차이를 보이 는 것으로 나타났다. 또한 집단 간에 유의미한 차이를 나타내 주는 시선추적 과제의 측정치는 고정 수, 고정 지속시간, 도약수, 회귀 수 등이었다. 도약 진폭에서는 집단 간에 유의미한 차이가 나타나지 않았다.

본 연구의 결과에 따른 논의는 다음과 같다.

시선추적장치를 활용한 읽기 평가에서 텍스트 처리의 특성이 읽 기장애 아동과 일반아동 간에 전반적으로 유의미한 차이를 나타 냈다. 이러한 결과는 첫째, 시선추적 연구의 과제 유형과 관련 지어 생각해볼 수 있다. 시선추적 연구는 읽기의 복잡성을 반영하여 언 어적이나 비언어적 과제를 통해 읽기의 양상을 측정하는 경향이 있 다. Bucci, Nassibi, Gerard, Bui-Quoc과 Seassau (2012)에 의하면 시각적 탐색 및 처리과제는 한 가지 목표물을 확인하거나 개수를 세고, 모든 글자들을 다 봐야 하기 때문에 생략이나 도약이 가능한 읽기 과제보다 과제를 수행하기에 더 어려울 수 있다고 한다. 또한 Kirkby 등(2011)은 두 과제에 대한 인지적 요구 수준이 다르고, 읽 기장애 아동들의 대다수가 안구의 협응 문제를 경험하는 것은 아 니며, 인과관계보다는 상관관계로 해석할 필요가 있고, 읽기의 어 려움이 오히려 안구운동의 비전형적인 특성을 유발한다고 하였다. 실제로 시각적 탐색이나 처리 과제에서 읽기장애 집단과 일반아동 집단 간에 유의미한 차이가 없다는 연구결과들이 많이 있다(De Luca et al., 1999; Kirkby et al., 2011; Prado et al., 2007). 이러한 점 을 감안할 때 시선추적 연구에서 정상군과 장애군의 읽기 특성을 살펴보기 위해서는 언어적으로 과제를 제시하여 측정하면 더 타당 할 것이라고 본다. 둘째, 언어적 과제의 경우에도 자극을 어느 수준 으로 제시할 것인지를 고려할 필요가 있다. 낱말보다는 문장 이상 의 수준으로 제시할 경우 낱말이나 구, 문장, 텍스트 수준에서 상호 간의 관련성을 볼 수 있어 전반적인 읽기능력을 평가할 수 있도록 해 주고, 낱말 수준보다 눈의 움직임의 다양한 측면을 분석할 수 있 다. 또한 텍스트 처리를 하는 동안 눈의 움직임을 통해 피험자의 연 령, 읽기능력, 지식 등에 따라 다양한 수행능력을 측정할 수 있다 (Kaakinen \& Hyönä, 2007; Raney et al., 2014; Rayner, 1978). 따라 서 측정된 결과를 바탕으로 텍스트 수준에서 읽기장애 아동과 일 반아동의 읽기 양상을 비교해 볼 수 있다.

한편 두 집단의 텍스트 처리 양상에 관한 효과 크기는 전체적으 로 유의미하게 나타났으나 개별 연구의 효과크기를 살펴보면 12개 의 연구 중에서 4 편은 유의미하지 않은 것으로 나타났다(De Luca et al., 1999; Hatzidaki et al., 2011; Kirkby et al., 2011; Vagge, Cavanna, Traverso, \& Iester, 2015). 이들 4편에 대해 구체적으로 살펴보면 첫째, 표본의 수가 비교적 작은 편이라고 볼 수 있다. 즉 읽기장애 아동 집단의 경우 4 편의 평균표본 수는 11.25 명으로 다른 8 편의 평 균표본수인 20.25 명에 비해 작은 편이었다. 일반아동의 경우에도 4 편의 평균표본 수는 10.75 명으로 다른 8 편의 평균표본수인 19.75 명 에 비해 작은 것으로 나타났다. 따라서 이들 4 편의 작은 표본 수가 효과 크기에 영향을 준 것으로 볼 수 있다. 특히 Kirkby 등(2011)의 연구는 표본 수가 8 명에 불과하여 연구 전체뿐만 아니라 2 개의 종 속변수(고정 수, 고정 지속시간) 모두에 대해서도 효과 크기가 유의 미하지 않게 나타났다. 둘째, 시선추적 연구의 측정치, 즉 종속변수 의 측면에서 4 편 중 2 편이 도약 진폭을 측정하였다(De Luca et al., 1999; Hatzidaki et al., 2011). 본 연구결과에 따르면 측정치들 중에 서 유일하게 도약 진폭만이 집단 간의 차이를 유의하게 나타내지 않는 변수로 나타났다(Table 3, Figure 7). 이러한 종속변인의 특성 이 연구의 전체 효과크기에 반영된 것으로 볼 수 있다.

시선추적 연구의 측정치들 중에서 집단 간 텍스트 처리의 양상 을 유의미하게 구별해주는 변수들은 고정 수, 고정 지속시간, 도약 수, 그리고 회귀 수로 나타났다. Table 3을 보면 각 측정치들의 효과 크기가 제시되어 있는데, 고정 지속시간, 도약 수, 고정 수, 회귀 수 의 순으로 효과크기가 큰 것으로 나와 있다. 그 중에서도 고정 지속 시간은 본 연구에서 채택한 12 개의 논문 중에서 8 편에 의해 종속변 수로 다루어진 점을 감안할 때, 텍스트 처리에 있어 두 집단을 변별 하는 대표적인 변수라 할 수 있다. De Luca 등(1999)도 고정 지속시 간이 도약과 더불어 읽기장애 측정에서 신뢰할 만한 변수라고 했 다. Figure 5에 제시된 개별 연구들의 고정 지속시간에 대한 효과크 기는 Kirkby 등(2011)의 연구를 제외하고는 모두 유의미하게 나타 났다. 그러나 Kirkby 등(2011)의 연구도 집단별로 8 명이라는 작은 표본 수에 의해 고정 지속시간의 효과 크기가 유의미하게 나타나지 않았을 가능성이 있다. 또한 Hutzler와 Wimmer (2004)에 의하면 개별 연구에서 사용한 언어의 특성에 따라 고정 지속시간에 차이 가 있다고 했다. 즉, 독일어는 영어의 음절구조와 비슷하지만 이탈 리아어와는 달라서 독일의 읽기장애 아동은 이탈리아의 읽기장애 아동보다 좀 더 긴 고정 지속시간을 나타낸다고 했다. 이는 시선추 적 연구가 언어별 읽기장애 집단의 텍스트 처리 특성도 분석할 수 있다는 점을 시사해준다.

한편 본 연구에서 메타분석을 한 결과, 도약 진폭은 집단 간의 차 이를 유의미하게 나타내주는 측정치가 아닌 것으로 나타났다. 도약 진폭은 시선추적 연구의 다른 측정치들과는 달리 읽기장애 아동 에 비해 일반아동에게서 더 크게 나타나는 경향이 있다. 그런데 본 
연구결과, Bucci 등(2012)과 Razuk, Barela, Peyre, Gerard와 Bucci (2018)에서는 부적인 효과크기를 나타냄으로써 전체적인 효과크 기를 상쇄시켜 유의미한 효과크기가 나타나지 않은 것으로 볼 수 있다(Figure 7). 이와 같이 도약 진폭에 대해서 일관된 효과크기 패 턴이 나타나지 않았다는 연구결과를 볼 때, 아동의 읽기능력을 평 가함에 있어서 대상자의 특징을 파악할 수는 있으나, 선별을 위한 효율적인 기준이 되기는 어려움을 시사한다. 또한 도약 진폭에 대 한 효과크기가 일관성 있게 나타나지 않은 것은 읽기장애 아동 집 단의 이질성에서 비롯되었을 가능성이 있다. 따라서 본 연구에 선 정된 논문 중에서 도약 진폭을 종속변수로 한 논문이 6편밖에 되 지 않아 읽기장애 아동의 도약 진폭의 특성을 파악하기 위해서는 더 많은 수의 후속연구가 필요할 것으로 보인다.

본 메타분석 연구를 통해 시선추적장치를 활용한 읽기 평가가 읽기장애 아동과 일반아동의 텍스트 처리 특성을 변별하는 데 유 용한 도구임을 확인하였다. 또한 시선추적장치의 측정치에 따라서 두 집단을 구별하는 효과크기의 정도가 다르다는 것을 확인하였 다. 읽기장애 아동의 선별을 위해서는 일관성 있고, 효과크기가 높 은 측정치를 사용할 필요가 있으며, 이는 읽기 아동의 조기 선별에 도움을 줄수 있다고 본다.

본 연구의 제한점 및 제언은 첫째, 읽기장애 아동 집단의 이질성 을 고려하여 읽기장애 아동을 유형이나 수준별로 나누어 각 측정 치에 따른 읽기 특성을 살펴볼 필요가 있다. 둘째, 각 연구에서 사 용된 읽기 평가는 묵독이나 음독 과제로 구분되어 실시되었으며, 텍스트의 난이도 또한 수준을 달리하여 자극 과제로 제시하기도 하였다. 따라서 후속연구에서는 과제의 유형이나 텍스트의 난이도 에 따른 분석을 통해 이들이 효과크기에 어떠한 영향을 미치는지 를 살펴볼 필요가 있다. 셋째, 언어에 따른 분석을 통해 언어별 특성 이 각 측정치에 대한 효과크기에 어떻게 반영되는지를 살펴볼 필요 가 있다. 이를 통해 시선추적장치를 활용하여 장애 아동의 선별 또 는 중재 효과를 확인함에 있어서 언어적 특수성을 고려할 필요가 있는 이중언어 사용자나 다문화 가족 구성원들의 읽기평가에도 기 여할 수 있을 것으로 본다.

\section{REFERENCES}

Bucci, M. P., Nassibi, N., Gerard, C. L., Bui-Quoc, E., \& Seassau, M. (2012). Immaturity of the oculomotor saccade and vergence interaction in dyslexic children: evidence from a reading and visual search study. PloS One, 7, e33458.

Choi, S. K. (2013). The exploring reading education for multimodal text based reading behavior analysis in digital literacy environment. Journal of Reading Research, 29, 225-264.

Choi, W., Lowder, M. W., Ferreira, F., Swaab, T. Y., \& Henderson, J. M. (2017).

Effects of word predictability and preview lexicality on eye movements during reading: a comparison between young and older adults. Psychology and Aging, 32, 232-242.

Chung, B. J., Kim, Y. T., \& Yoon, H. J. (2017). Relative contributions of components to different reading comprehension tasks in grade 3-4. Communication Sciences \& Disorders, 22, 66-75.

De Luca, M., Borrelli, M., Judica, A., Spinelli, D., \& Zoccolotti, P. (2002). Reading words and pseudowords: an eye movement study of developmental dyslexia. Brain and language, 80, 617-626.

De Luca, M., Di Pace, E., Judica, A., Spinelli, D., \& Zoccolotti, P. (1999). Eye movement patterns in linguistic and non-linguistic tasks in developmental surface dyslexia. Neuropsychologia, 37, 1407-1420.

Dürrwächter, U., Sokolov, A. N., Reinhard, J., Klosinski, G., \& Trauzettel-Klosinski, S. (2010). Word length and word frequency affect eye movements in dyslexic children reading in a regular (German) orthography. Annals of Dyslexia, 60, 86-101.

Gersten, R., Fuchs, L. S., Compton, D., Coyne, M., Greenwood, C., \& Innocenti, M. S. (2005). Quality indicators for group experimental and quasiexperimental research in special education. Exceptional Children, 71, 149164.

Habib, M. (2000). The neurological basis of developmental dyslexia: an overview and working hypothesis. Brain, 123, 2373-2399.

Hatzidaki, A., Gianneli, M., Petrakis, E., Makaronas, N., \& Aslanides, I. M. (2011). Reading and visual processing in Greek dyslexic children: an eyemovement study. Dyslexia, 17, 85-104.

Hutzler, F., \& Wimmer, H. (2004). Eye movements of dyslexic children when reading in a regular orthography. Brain and Language, 89, 235-242.

Hyönä, J., \& Olson, R. K. (1995). Eye fixation patterns among dyslexic and normal readers: effects of word length and word frequency. Journal of Experimental Psychology: Learning, Memory, and Cognition, 21, 1430-1440.

Kaakinen, J. K., \& Hyönä, J. (2007). Perspective effects in repeated reading: an eye movement study. Memory \& Cognition, 35, 1323-1336.

Kirkby, J. A., Blythe, H. I., Drieghe, D., \& Liversedge, S. P. (2011). Reading text increases binocular disparity in dyslexic children. PLoS One, 6, e27105.

Kwon, N., \& Sturt, P. (2014). The use of control information in dependency formation: an eye-tracking study. Journal of Memory and Language, 73, 59-80. 
Lee, S. R., \& Suh, H. (2013). The current status and perspectives of research on reading process using eye-tracker. Korean Language Education Research, 46, 479-503.

Martos, F. J., \& Vila, J. (1990). Differences in eye movements control among dyslexic, retarded and normal readers in the Spanish population. Reading and Writing, 2, 175-188.

Pavlidis, G. T. (1981). Do eye movements hold the key to dyslexia? Neuropsychologia, 19, 57-64.

Prado, C., Dubois, M., \& Valdois, S. (2007). The eye movements of dyslexic children during reading and visual search: impact of the visual attention span. Vision Research, 47, 2521-2530.

Raney, G. E., Campbell, S. J., \& Bovee, J. C. (2014). Using eye movements to evaluate the cognitive processes involved in text comprehension. Journal of Visualized Experiments, (83), e50780.

Rayner, K. (1978). Eye movements in reading and information processing. Psychological Bulletin, 85, 618-660.

Rayner, K. (1985). Do faulty eye movements cause dyslexia? Developmental Neuropsychology, 1, 3-15.

Razuk, M., Barela, J. A., Peyre, H., Gerard, C. L., \& Bucci, M. P. (2018). Eye movements and postural control in dyslexic children performing different visual tasks. PloS One, 13, e0198001.

Rommelse, N. N., Van der Stigchel, S., \& Sergeant, J. A. (2008). A review on eye movement studies in childhood and adolescent psychiatry. Brain and Cognition, 68, 391-414.

Seassau, M., Gérard, C. L., Bui-Quoc, E., \& Bucci, M. P. (2014). Binocular saccade coordination in reading and visual search: a developmental study in typical reader and dyslexic children. Frontiers in Integrative Neuroscience, $8,85$.

Suh, H., Kim, J., Pyeon, J., \& Shin, Y. (2016). A research of characteristics and eye-tracking of readers in problem-solving context-focused on readers' task commitment according to the reading level. Journal of Reading Research, 38, 225-254.

Trauzettel-Klosinski, S., Koitzsch, A. M., Dürrwächter, U., Sokolov, A. N., Reinhard, J., \& Klosinski, G. (2010). Eye movements in German-speaking children with and without dyslexia when reading aloud. Acta Ophthalmologica, 88, 681-691.

Vagge, A., Cavanna, M., Traverso, C. E., \& Iester, M. (2015). Evaluation of ocular movements in patients with dyslexia. Annals of Dyslexia, 65, 24-32. 
Appendix 1. Summary of studies included in the meta-analysis

\begin{tabular}{|c|c|c|c|c|c|c|c|c|c|c|}
\hline \multirow[b]{2}{*}{ No. } & \multirow[b]{2}{*}{ Study } & \multicolumn{3}{|c|}{$\mathrm{RD}$ group } & \multicolumn{3}{|c|}{ Control group } & \multirow{2}{*}{$\begin{array}{l}\text { Native } \\
\text { language }\end{array}$} & \multirow[b]{2}{*}{ Task } & \multirow[b]{2}{*}{ Dependent measure } \\
\hline & & $\mathrm{N}$ & Age (yr) & $\begin{array}{c}\text { Gender } \\
\text { (boy:girl) }\end{array}$ & $\mathrm{N}$ & Age (yr) & $\begin{array}{c}\text { Gender } \\
\text { (boy:girl) }\end{array}$ & & & \\
\hline 1 & Bucci et al. (2012) & 12 & $11 \pm .6$ & NA & 9 & $11 \pm .9$ & NA & French & Silent reading & $\begin{array}{l}\text { Number of fixations, fixation duration, am- } \\
\text { plitude of saccades }\end{array}$ \\
\hline 2 & De Luca et al. (1999) & 10 & $12.6 \pm 2.0$ & $8: 2$ & 10 & $10.5 \pm 1.0$ & NA & Italian & Silent reading & $\begin{array}{l}\text { Number of saccades/line, number of re- } \\
\text { gressions/line, \% of regressions/line, } \\
\text { amplitude of saccades, amplitude of re- } \\
\text { gressions, fixation duration }\end{array}$ \\
\hline 3 & Hatzidaki et al. (2011) & 16 & $11.5 \pm 1.51$ & NA & 14 & $12.14 \pm .95$ & NA & Greek & Loud reading & $\begin{array}{l}\text { Number of fixations, total number of fixa- } \\
\text { tions, number of saccades/line, number } \\
\text { of regressions/line, fixation duration, } \\
\text { amplitude of saccades/line, amplitude of } \\
\text { regressions/line }\end{array}$ \\
\hline 4 & Hutzler \& Wimmer (2004) & 11 & $13.6 \pm .42$ & 11 & 11 & $13.3 \pm .33$ & 11 & German & Silent reading & $\begin{array}{l}\text { Number of fixations, fixation duration, \% } \\
\text { of regressions }\end{array}$ \\
\hline 5 & Hyönä \& Olson (1995) & 21 & 14.4 & NA & 21 & 10.5 & NA & English & Loud reading & $\begin{array}{l}\text { Number of first pass fixation (word), total } \\
\text { number of duration (word), first fixation } \\
\text { duration (word), gaze duration (word) }\end{array}$ \\
\hline 6 & Kirkby et al. (2011) & 8 & $11.38 \pm 1.1$ & NA & 8 & $10.67 \pm 1.1$ & NA & English & Silent reading & $\begin{array}{l}\text { Fixation duration, saccade length, regres- } \\
\text { sion frequency, number of fixations }\end{array}$ \\
\hline 7 & Martos \& Vila (1990) & 30 & $7-14$ & $21: 9$ & 30 & $7-14$ & $21: 9$ & Spanish & Silent reading & $\begin{array}{l}\text { Number of saccades, saccadic fixation du- } \\
\text { ration, number of regressions, regressive } \\
\text { fixation duration }\end{array}$ \\
\hline 8 & Prado et al. (2007) & 14 & $11.08 \pm 1.0$ & $11: 3$ & 14 & $10.66 \pm .61$ & $9: 5$ & French & Loud reading & $\begin{array}{l}\text { Total number of fixations, mean fixation } \\
\text { duration, number of saccadic fixations, } \\
\text { saccadic fixation duration, number of re- } \\
\text { gressive fixations, } \% \text { of regressive (left- } \\
\text { ward) fixations, regressive fixation dura- } \\
\text { tion }\end{array}$ \\
\hline 9 & Razuk et al. (2018) & 15 & $9.8 \pm 1.3$ & $13: 2$ & 15 & $10.0 \pm 1.3$ & $13: 2$ & French & Silent reading & $\begin{array}{l}\text { Fixation duration, amplitude of pro-sac- } \\
\text { cades }\end{array}$ \\
\hline 10 & Seassau et al. (2014) & 43 & $10.6 \pm 1.6$ & NA & 42 & $10.7 \pm 1.5$ & NA & French & Silent reading & $\begin{array}{l}\text { Number of fixations, fixation duration, } \\
\text { number of saccades, amplitude of sac- } \\
\text { cades, number of regressions, amplitude } \\
\text { of regressions }\end{array}$ \\
\hline 11 & $\begin{array}{l}\text { Trauzettel-Klosinski et al. } \\
(2010)\end{array}$ & 16 & $9.5 \pm .3$ & $12: 4$ & 16 & $9.6 \pm .4$ & 10:6 & German & Loud reading & $\begin{array}{l}\text { Number of words/min, number of sac- } \\
\text { cades/word, amplitude of saccades, \% } \\
\text { of regressions, fixation duration, number } \\
\text { of regressions/word, number of fixa- } \\
\text { tions/word }\end{array}$ \\
\hline 12 & Vagge et al. (2015) & 11 & $9.4 \pm 1.6$ & $7: 4$ & 11 & $9.2 \pm 1.5$ & $6: 5$ & Italian & Silent reading & $\begin{array}{l}\text { Number of saccades, number of saccades/ } \\
\text { word, number of syllables/sec, number } \\
\text { of regressions }\end{array}$ \\
\hline
\end{tabular}

Values are presented as mean $\pm S D$ or range.

$\mathrm{RD}=$ reading disability; $\mathrm{NA}=$ not available. 


\section{국문초록}

\section{시선추적장치를 활용한 읽기장애 아동의 텍스트 처리 특성에 관한 메타분석}

김정아 · 오세진 · 최은정 · 김영태 · 성지은

이화여자대학교 언어병리학과

배경 및 목적: 읽기에 문제가 있는 아동들은 학업능력이 떨어지거나 사회적으로 상호작용을 하는 데 어려움을 겪을 가능성이 높다. 따 라서 읽기 평가를 통해 장애아동을 조기에 선별하는 것이 중요하다. 시선추적 과제는 읽기 평가에 유용한 도구로 알려져 있다. 본 연구 에서는 시선추적장치를 활용한 선행연구들을 고찰하여 텍스트 처리 수준에서 읽기장애 아동을 변별할 수 있는지를 살펴보고, 시선추 적 과제의 어떤 변수에서 집단 간에 유의한 차이가 나타나는지를 분석하고자 하였다. 방법: 9 개의 국내외 데이터베이스(Academic Search Complete, PsycINFO, ERIC, PubMed, CINAHL Plus, ScienceDirect, RISS, DBpia, Kyobo Scholar)를 통해 포함 및 제외 기준을 적용하여 총 12 편의 문헌을 분석대상으로 선정하여, Hedges' $g$ 를 사용하여 효과크기를 산출하였다. 결과: 시선추적 과제에 대한 메타 분석 결과, 읽기장애 아동 집단과 일반아동 집단은 유의한 차이를 보였다. 집단 간의 차이를 유의하게 나타낸 시선추적 과제의 측정치 들은 고정 수, 고정 지속시간, 도약수, 회귀 수 등이었다. 그러나 도약 진폭은 집단 간에 유의미한차이를 나타내 주지 않았다. 논의 및 결 론: 텍스트 처리에 대한 시선추적 과제가 읽기장애 집단을 변별하는 데 유용한 도구임을 확인할 수 있었으며, 이는 앞으로 읽기장애를 조기에 선별하는 데 활용될 수 있음을 시사한다.

핵심어: 시선추적, 읽기장애, 텍스트 처리, 메타분석

본 논문은 대한민국 교육부와 한국연구재단의 지원을 받아 수행된 연구임(No. NRF-2015S1A5A2A03049681).

\section{참고문헌}

서혁, 김지희, 편지윤, 신윤하(2016). 문제 해결 상황에서 독자의 눈동자 움직임 및 뇌파 특성 분석. 독서연구, 38, 225-254.

이소라, 서혁(2013). 시선추적장치를 활용한 읽기과정 연구의 현황과 가능성 탐색. 국어교육학연구, 46, 481-503.

정부자, 김영태, 윤효진(2017). 읽기이해검사의 형식에 따른 관련요인탐색: 초등학교 3-4학년을 대상으로. 언어청각장애연구, 22, 66-75.

최숙기(2013). 복합 양식 텍스트에 대한 독자의 읽기 행동 분석에 기반한 디지털 시대의 읽기 교육 방안 탐색. 독서연구, 29, 225-264. 